\title{
Myth: glucagon is an effective first-line therapy for esophageal foreign body impaction
}

Sanjay Arora, MD; Peter Galich, MD

\section{Introduction}

Esophageal foreign body impactions (EFBIs) are relatively rare in emergency medicine; patients rarely have them more than once. Indications for watchful waiting include a patent airway and the ability to clear secretions. However, in many cases, significant discomfort combined with patient expectations mandate early intervention to dislodge the foreign body. Endoscopy is the preferred definitive method of intervention for EFBI; however, most emergency physicians choose to apply one of several less invasive modes of therapy as firstline treatment. Pharmacologic agents that have been described for this purpose include glucagon, nifedipine, sublingual nitroglycerin, proteolytic enzymes and benzodiazepines. Among these, glucagon has become the most widely accepted in clinical practice.

Glucagon acts by relaxing the smooth musculature of the lower esophagus, thereby reducing the resting pressure of the lower esophageal sphincter., ${ }^{1,2}$ Thus esophageal obstructions at the level of the lower esophageal sphincter (T10), which is the third most common site of obstruction in the esophagus, may be alleviated by glucagon..$^{3-5}$ Despite this hypothetical advantage, there is no high-quality data to support the use of glucagon for EFBI. In this paper, we review the published literature on this topic to illustrate the myth that glucagon is an effective first-line therapy for EFBI.

\section{Methods}

A literature search was performed through MEDLINE and PubMed using the keywords "glucagon" and "esophageal food impaction." All abstracts were reviewed for relevance, and articles were retrieved that evaluated the effectiveness of glucagon for the treatment of EFBI. To ensure that all relevant articles were included in this paper, we performed an ancestral search on all the aforementioned key articles.

\section{Literature}

The ancestral search revealed that intravenous administration of glucagon was first suggested as a method of treating EFBI in 1977. ${ }^{6}$ This was based on the then-recent discovery that glucagon had a direct relaxing effect on lower esophageal sphincter tone. This first study showed some promise, and several small case reports and subsequent case series reported success rates between $37 \%$ and $100 \% .^{7-9}$

In 1995, Tibbling and colleagues ${ }^{10}$ published a multicentre, placebo-controlled, double-blind study comparing the concurrent use of glucagon and diazepam with placebo in patients with EFBI. Of the 43 patients enrolled with this condition, 24 were randomized to the treatment group and 19 to the placebo group. Alleviation of the impaction was seen in 9 of 24 (38\%) patients in the glucagon and diazepam group, compared with 6 of $19(32 \%)$ in the placebo group, a difference that was

From the Department of Emergency Medicine, Keck School of Medicine, University of Southern California, Los Angeles, Calif.

Submitted Apr. 26, 2008; Accepted Sep. 16, 2008

This article has not been peer reviewed.

CJEM 2009;11(2):169-71 
not statistically significant. However, it is important to note that the study had several significant limitations. First, the small sample size meant the study was underpowered to detect a difference, if one exists. Second, the treatment group received both glucagon and diazepam, thus affecting both smooth and striated muscle. If a treatment effect does exist with glucagon in isolation, it is impossible to detect it with this study design. Finally, the study excluded all patients with known esophageal strictures. This exclusion criterion likely inflated the success rate of both the treatment and placebo groups by selecting cases without underlying esophageal pathology. Moreover, in the emergency department, medical history is frequently challenging to obtain, making it difficult for the clinician to select patients without esophageal stricture for pharmacologic treatment. Thus the only real conclusion that can be drawn from this study is that the combination of glucagon and diazepam was not more effective than placebo in this small, specified patient group.

In 2001, Mehta and coworkers ${ }^{11}$ conducted a prospective randomized, double-blinded, placebo-controlled 2-phase study using glucagon for pediatric patients with coin ingestions. A total of 14 children between the ages of 1 and 8 years who had radiographically confirmed esophageal coin ingestions were enrolled. In the first phase, the patients were randomized to receive either weight-based glucagon or placebo. A success rate of 2 of $15(13 \%)$ was observed in the treatment group versus 3 of $5(60 \%)$ in the placebo group. The second phase was open-label and patients in either group with persistent EFBI could receive an open-label glucagon dose at the discretion of the treating physican. Six patients received an additional dose of glucagon because the first dose was ineffective. None of these extra doses resulted in passage of the coin. This study failed to show a significant difference between the groups, but was underpowered to do so. In this study, 11 of the 14 subjects had an EFBI in the upper third of the esophagus. Since glucagon acts primarily on the smooth muscle of the lower esophagus, the result may have been different if only patients with lower EFBI had been enrolled. This study, although small, was notable for the fact that a large proportion of the treatment group experienced vomiting $(11 / 15,73 \%)$. In fact, 1 patient was removed from the study because of chest pain that may have been a result of vomiting. Vomiting is a well-known side effect of glucagon and can result in aspiration and morbidity.

In 2004, Sodeman and associates ${ }^{12}$ conducted a singlecentre, retrospective case series of 222 cases of EFBI, of which 106 (48\%) received glucagon. Of those 106 pa- tients, $10(9 \%)$ had complete resolution of symptoms, versus 20 of $116(17 \%)$ whose symptoms resolved spontaneously without a pharmacologic adjunct. Because of the selection bias inherent in this observational study, and its retrospective design, it is difficult to draw conclusions about the efficacy of glucagon versus no treatment, as we have no insight into why some patients received glucagon while others did not.

Despite this limitation, the success rate of glucagon for treatment of EFBIs in this study was the lowest of the 4 studies reviewed and does not support its use. This study also notes that fixed esophageal narrowing and meat impactions are less likely to be alleviated by noninvasive techniques. Therefore, the utility of this study may be in the clinical guidance it provides toward nonpharmacologic intervention in the subset of patients with known esophageal pathology or a meat impaction.

In 2006, Al-Haddad and colleagues ${ }^{13}$ published a retrospective case series of 92 patients with suspected EFBI, all of which were caused by food. Glucagon was administered in all cases; 30 of 92 patients (33\%) had complete resolution of symptoms, and 62 of 92 patients (67\%) did not and underwent endoscopy. Because of its retrospective design, this study had many limitations, with the primary one being selection bias. The number of patients who went home without receiving glucagon and why the enrolled patients received glucagon are not reported. Also, there was no placebo group for comparison. In the discussion, the authors note that their observed resolution rate was no better than placebo in the aforementioned Tibbling study. ${ }^{10}$ Also, because of the noncontrolled design, there is no way of knowing if it was the glucagon and not any of the other medications the patients may have received that resulted in resolution. Of the 92 patients, 19 received benzodiazepine (diazepam or lorazepam), in addition to glucagon at the discretion of the emergency physician. In this subset of patients, 11 of 19 $(58 \%)$ had relief of symptoms, compared with 19 of 73 $(26 \%)$ of those who received glucagon alone $(p<0.01)$. Based on this data, the authors suggest that benzodiazepine (alone or in combination with glucagon) may aid in the relief of EFBI more efficaciously than glucagon alone.

\section{Conclusion}

The literature regarding the use of glucagon for EFBI lacks randomized, double-blinded controlled studies with the power necessary to detect efficacy differences. As discussed above, the largest randomized, double-blinded controlled study enrolled only 43 patients and employed the concurrent use of a benzodiazepine in addition to 
glucagon. Although the studies were small, none found a statistically significant advantage for the use of glucagon. In fact, glucagon may cause a reduction in the amplitude of esophageal contractions and blunt peristalsis causing a delay in esophageal transit time..$^{1,6,14,15}$ One author suggests that glucagon "may impede disimpaction" of EFBI. ${ }^{16}$ Intravenous glucagon is also expensive (about US\$70 per 1-mg vial). It is clear that glucagon commonly causes vomiting and therefore poses a risk of aspiration, esophageal perforation and increased morbidity. Without clear evidence that glucagon therapy improves the resolution of EFBI, coupled with the knowledge that it is costly and is likely to cause vomiting, it is our opinion that glucagon should not be used as a first-line agent for EFBI.

Competing interests: None declared.

Keywords: glucagon, esophageal foreign body, food bolus

\section{References}

1. Colon V, Grade A, Pulliam G, et al. Effect of doses of glucagon used to treat food impaction on esophageal motor function of normal subjects. Dysphagia 1999;14:27-30.

2. Hogan WJ, Dodds WJ, Hoke SE, et al. Effect of glucagon on esophageal motor function. Gastroenterology 1975;69:160-5.

3. Ratcliff KM. Esophageal foreign bodies. Am Fam Physician 1991;44:824-31

4. Maglinte DD, Chernish SM, Kelvin FM, et al. Pharmacoradiologic disimpaction of esophageal foreign bodies: review and recommendation. Emerg Radiol 1995;2:151-7.

5. Lacy PD, Donnelly MJ, McGrath JP, et al. Acute food bolus impaction: aetiology and management. J Laryngol Otol 1997; 111:1158-61.

6. Ferrucci JT Jr, Long JA Jr. Radiologic treatment of esophageal food impaction using intravenous glucagon. Radiology 1977; $125: 25-8$

7. Trenkner SW, Maglinte DDT, Lehman GA, et al. Esophageal food impaction: treatment with glucagon. Radiology 1983;149: 401-3.

8. Bryant BG, Trout DJ. Treatment of esophageal food impaction a new use for glucagon. Drug Intell Clin Pharm 1982;16:407-9.

9. Reddy AN. Noninvasive management of esophageal meat impaction. Gastrointest Endosc 1981;27:202-3.

10. Tibbling L, Bjorkhoel A, Jansson E, et al. Effect of spasmolytic drugs on esophageal foreign bodies. Dysphagia 1995;10:126-7.

11. Mehta DI, Attia MW, Quintana EC, et al. Glucagon use for esophageal coin dislodgement in children: a prospective, double-blind, placebo-controlled trial. Acad Emerg Med 2001; 8:200-3.

12. Sodeman TC, Harewood GC, Baron TH. Assessment of the predictors of response to glucagon in the setting of acute esophageal food bolus impaction. Dysphagia 2004;19:18-21.

13. Al-Haddad M, Ward EM, Scolapio JS, et al. Glucagon for the relief of esophageal food impaction: Does it really work? Digestive Diseases and Sciences 2006;51:1930-3.

14. Channer KS, Bolton R, al-Hilli S, et al. The effect of glucagon on the swallowing of capsules. Br J Clin Pharmacol 1983;16: 456-8.

15. Anvari M, Richards D, Dent J, et al. The effect of glucagon on esophageal peristalsis and clearance. Gastrointest Radiol 1989; 14:100-2.

16. Fass R, Dekel R. Intravenous glucagon in food impaction Use it or lose it? Dysphagia 2004;19:15-7.

Correspondence to: Dr. Sanjay Arora, Department of Emergency Medicine, LAC + USC Medical Center, 1200 N. State St. Rm. 1011, Los Angeles CA 90033; sanjayar@usc.edu 